\title{
Simulations of small-pore microchannel plates for fast-gated $x$-ray imaging and spectroscopy of high-energy density plasmas
}

Craig A. Kruschwitz and Ming Wu

National Security Technologies, LLC

Los Alamos, New Mexico 87544

Greg Rochau

Sandia National Laboratories

Albuquerque, New Mexico 87185

June 2010 


\section{Abstract}

This poster describes work done at National Security Technologies, LLC (NSTec) and Sandia National Laboratories (SNL) over the past several years on the design and characterization of microchannel plate (MCP)-based fast-gated $x$-ray imagers for use on the SNL Z machine. These cameras use 10-micron-pore MCPs similar to the type used for spectroscopy and imaging applications at other facilities. To aid in the understanding of MCP behavior, we have developed a Monte Carlo simulation model for prediction of MCP response. The code contains a detailed physical model of the electron cascade and amplification process of the MCP that includes energy conservation for the secondary electrons, the effects of elastic scattering of low-energy electrons from the channel wall, and gain saturation mechanisms from wall charging and space charge. Our model can simulate MCP response for both static and pulsed voltage waveforms. Excellent agreement between the Monte Carlo simulations and laboratory measurements has been achieved. Here, we apply our simulation model to 2-micron-pore MCPs, which, while readily available from a variety of vendors, are not used in imaging applications. We investigated the DC and pulsed gain characteristics of such an MCP, with particular emphasis on dynamic range, temporal response, and spatial resolution. The results are compared to the predicted and measured characteristics of 10-micron-pore MCPs. 


\section{Introduction}

- There is a growing interest in the high-energy density physics (HEDP) and inertial confinement fusion communities to develop gated imaging diagnostics with spatial resolutions down to 5 microns and time resolutions down in the tens of picoseconds.

- Small-pore MCPs, while available from a variety of vendors, have not yet been used in HEDP imaging devices.

- Using a Monte Carlo code developed at NSTec over the past several years in collaboration with SNL, we investigated the potential performance of 2-micron-pore MCPs (the smallest pore size available) for HEDP imaging diagnostics.

- We found that the 2-micron-pore MCPs show some promise for achieving shorter gate times and higher spatial resolutions. 


\section{Monte Carlo Simulation Model}

- The Monte Carlo model describes the cascade and dynamics of electrons in a single microchannel.

- The cascade is taken to be initiated by some number of initial electrons determined by the user.

- Standard equations give the mean secondary emission yield for each primary electron as a function of incident energy and angle relative to the surface normal.

- Initial energy and direction of emitted secondary electrons are sampled from appropriate probability distributions.

- The trajectories of the secondary electrons are then calculated using straightforward nonrelativistic equations of motion.

- With the electron equations of motion solved, the impact energy and angle are determined, and thereby the initial conditions for the next generation of electrons.

- The code also includes elastic "reflection" of low-energy electrons from the channel walls, which is important for low voltages.

- Space charge and wall charging algorithms describe the saturation behavior in the channel. 


\section{Secondary Electron Emission Equations}

Secondary emission is a function of primary electron energy and impact angle. A few equations are used to determine the secondary yield. The following equations give the maximum average yield for incident angle, $\theta_{i}$, and the incident primary energy giving the highest average secondary electron yield, respectively

$$
\begin{aligned}
& \delta_{m}\left(\theta_{i}\right)=\delta_{m}(0)\left(1+\alpha \frac{\theta^{2}}{2 \pi}\right) \\
& V_{m}\left(\theta_{i}\right)=V_{m}(0)\left(1+\alpha \frac{\theta^{2}}{2 \pi}\right)
\end{aligned}
$$

where $\delta_{m}(0)$ is the mean secondary yield at normal incidence, typically 3 to 4 for MCP glass, $V_{m}(0)$ is the primary electron energy giving maximum yield at normal incidence, and $\alpha$ is a constant with a value expected to be 0 to 2 .

The following equation is then used to give the average secondary yield

$$
\delta\left(V_{i}, \theta_{i}\right)=\delta_{m}\left(\theta_{i}\right)\left(\frac{V_{i}}{V_{m}\left(\theta_{i}\right)} \exp \left[1-\frac{V_{i}}{V_{m}\left(\theta_{i}\right)}\right]\right)^{s}
$$

where $s$ is an adjustable parameter chosen to fit the data, and $V_{i}$ is the primary electron energy.

Elastic reflection of low-energy electrons from the channel surface is also included. The energydependent reflection probability is given by:

$$
\delta_{e l}\left(V_{i}\right)=\frac{\left(\sqrt{V_{i}+V_{0}}-\sqrt{V_{i}}\right)^{2}}{\left(\sqrt{V_{i}+V_{0}}+\sqrt{V_{i}}\right)^{2}}
$$

where $V_{0}$ is an adjustable parameter.

Previous experimental work on secondary electron emission from channel plate glass are referenced to determine the approximate values of $\delta_{m}(0)$ and $V_{m}(0)$. Comparisons to experimental data for the specific MCPs being modeled determine the specific values of these constants, as well as $s, \alpha$, and $V_{0}$. 


\section{MCP Saturation}

\section{Space Charge}

- Compute approximate, local perturbations to the applied field.

- As the number of electrons increased, the perturbations become progressively larger.

- This reduces electron time of flight.

- Therefore, it impacts with less energy.

- This leads to a reduction in secondary emission yield.

\section{Wall Charging}

- The secondary emission cascade leaves a net positive charge on the channel surface.

- This leads to fields that can perturb the applied field and the trajectory of electrons in the pore. 


\section{Sensitivity Comparisons}

\section{0-micron and 2-micron-pore MCPs}
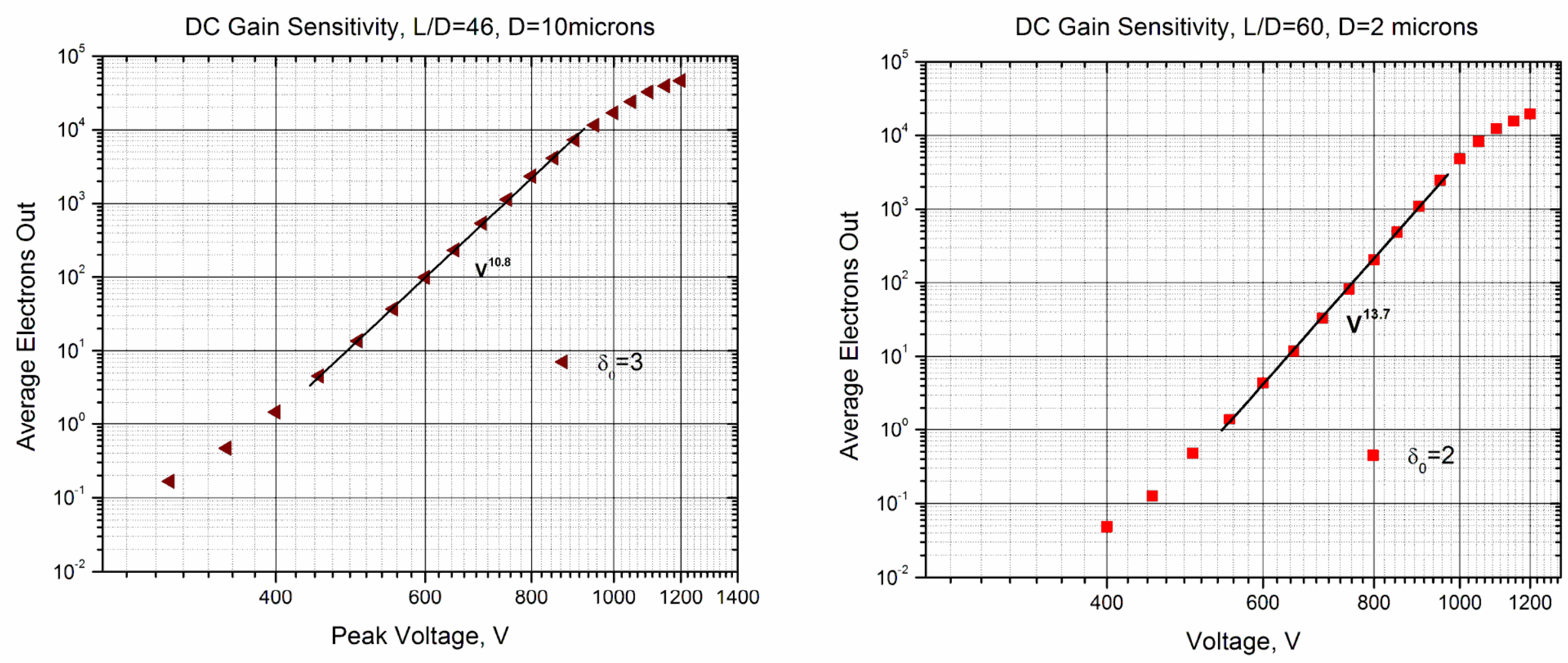

The DC gain curves of a 10-micron and 2-micron MCP are compared. The pore length to diameter ratio (L/D) are 46 and 60, respectively. As expected, the gain curve for the former is shallower than for the latter. Onset of nonlinearity occurs at smaller electron numbers in the 2-micron MCP, which is expected. 


\section{Electron Transit Time Comparison 10-micron and 2-micron-pore MCPs}

$L / D=46, D=10$ microns

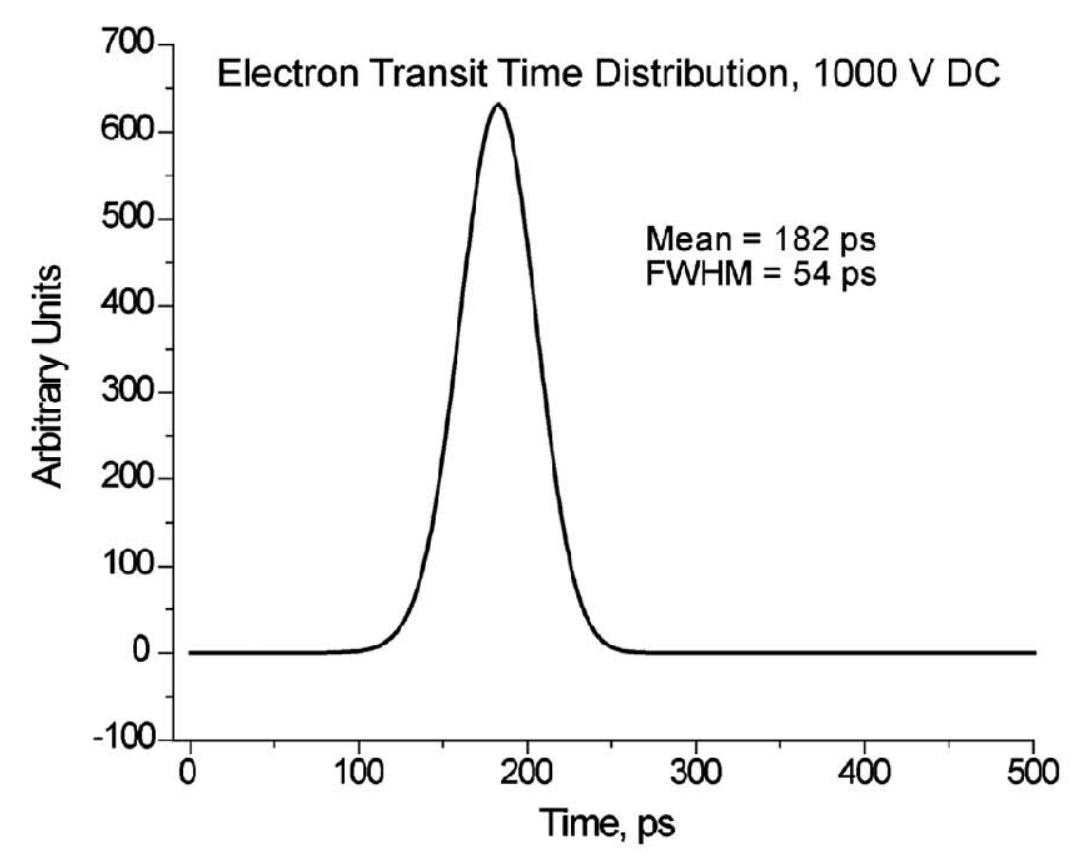

Electron Transit Time, $L / D=60, D=2$ microns

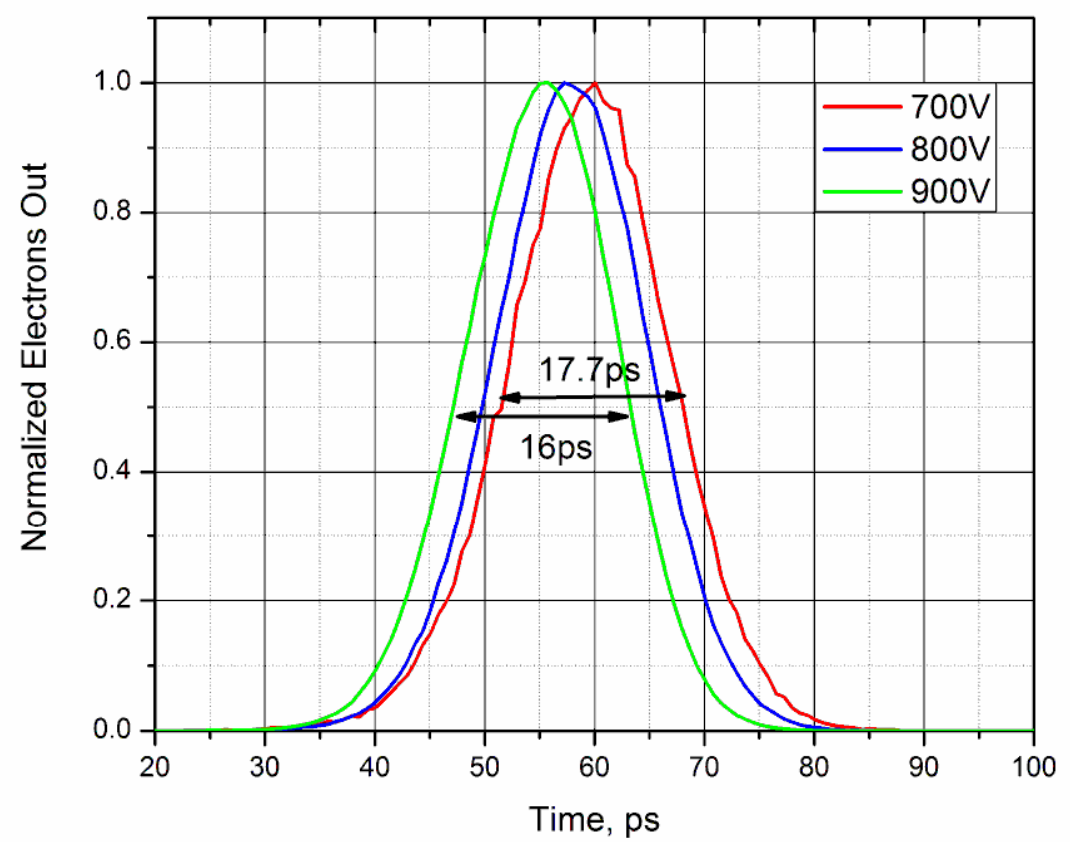

The electron transit time distribution for the 10-micron and 2-micron MCPs are compared. The 2-micron-pore MCP has a much smaller transit time (55 to $60 \mathrm{ps}$ for the given bias voltages vs $182 \mathrm{ps}$ for the slightly higher bias voltage on the 10-micron MCP) and smaller transit time spread (16 to 17.7 ps vs 54 ps for the 10-micron MCP). The transit time spread represents a theoretical limit on the possible time resolution from the MCP. 


\section{Gated MCP Perfomance: Simulated and Measured Gate Profiles 10-micron-pore MCP}

These data are for a gated x-ray imager using a 10-micron-pore, $\mathrm{L} / \mathrm{D}=46 \mathrm{MCP}$. The MCP has been coated with strips of conductive coating and a voltage pulse (shown later) sent across the strip, gating the MCP on. A femtosecond UV laser measures the gate profile of the MCP at different positions along the strip. The voltage pulse at various positions along the strip are also measured. These voltage pulse measurements are fed into the Monte Carlo code to simulate the gate profiles. There is excellent agreement between the measurements and the simulations.

\section{A Comparison between Experiment and Simulation Gate Profile Variation along the Strip}
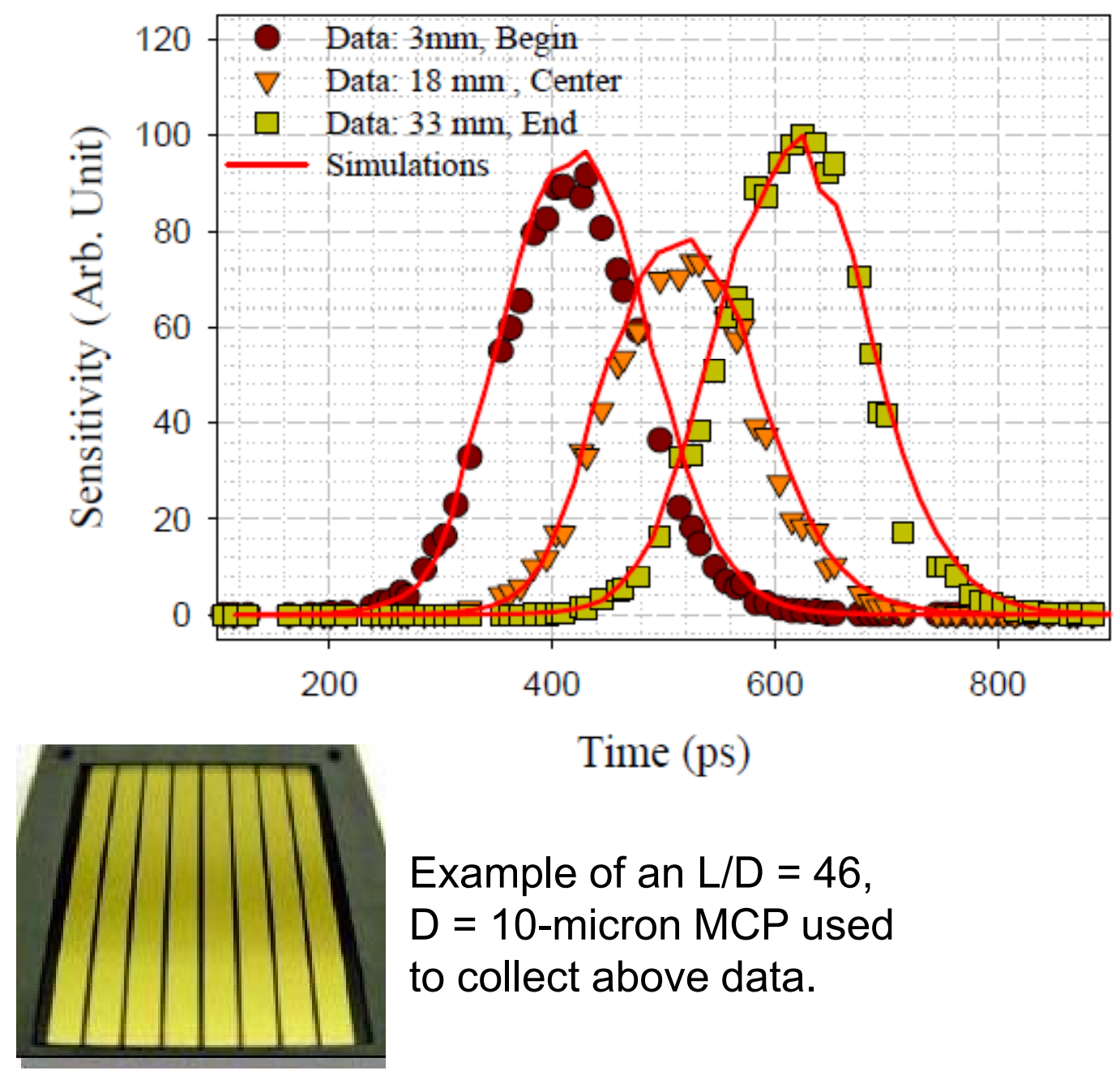

Time (ps)

Example of an $L / D=46$, $\mathrm{D}=10$-micron MCP used to collect above data. 


\section{Simulated Gate Profiles}

\section{2-micron MCP, L/D = 46}

Simulations were done using various voltage pulses; these are shown in the insets in the following plots. The voltage pulse shown in red is an actual pulse from a Kentech pulser, with a 150 ps flattop pulse-forming network. The other pulses are not real pulses, but are formed by cutting portions of the flat-top from the pulse. The shortest pulse is essentially just the rising and falling edge.

These voltage pulses were used to simulate the gate profile of a 2-micron MCP with various L/D ratios. These profile give an idea of the spatial resolutions one might

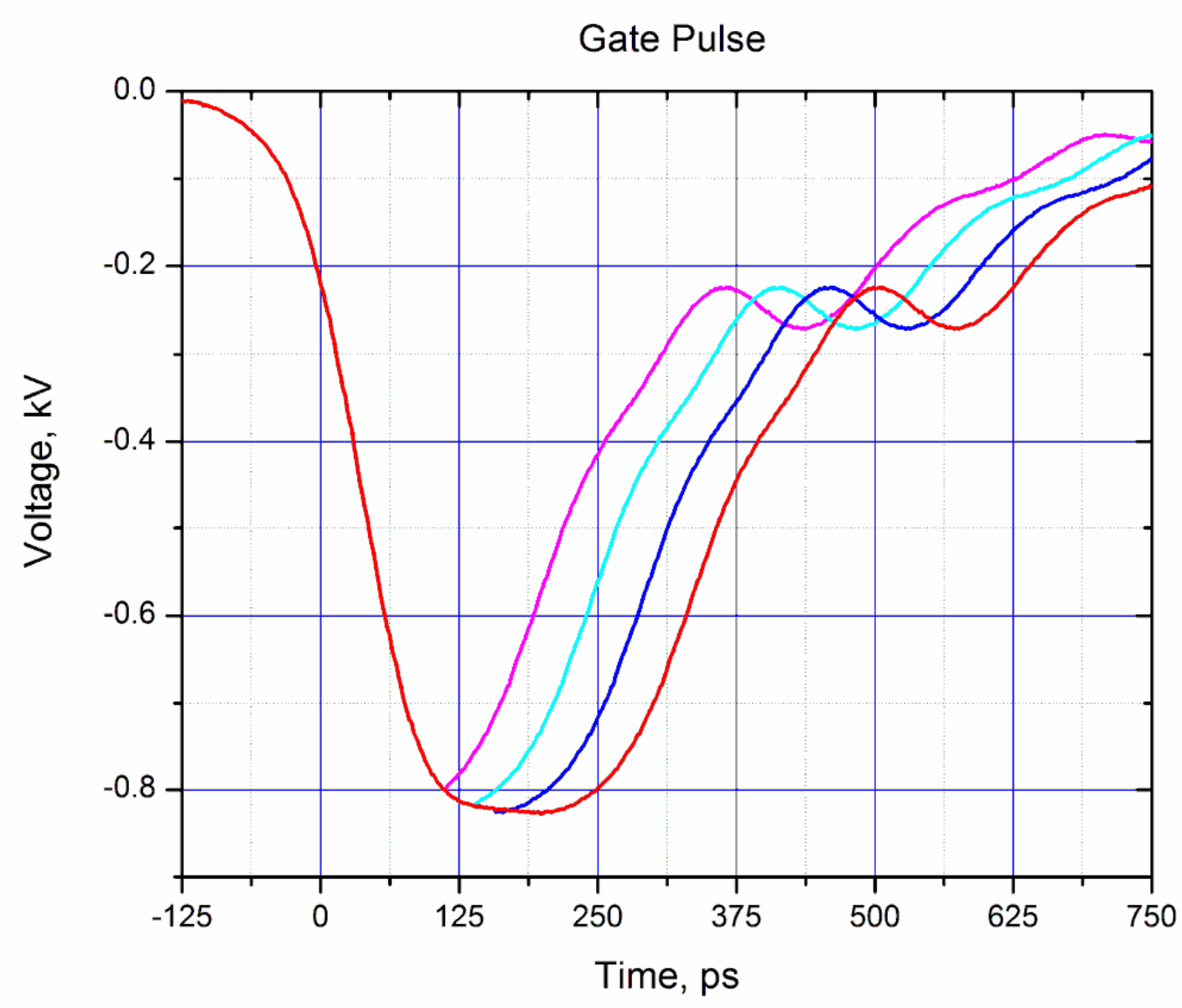
expect using a 2-micron-pore MCP as an imaging device. 


\section{Simulated Gate Profiles}

\section{2-micron MCP, L/D = 46}

The L/D $=46$ has gate profile widths (measured as $\mathrm{FWHM}$ ) ranging from about 67 ps up to 170 ps.

There is substantial gain, even for the shortest pulse (peak gain about 530).

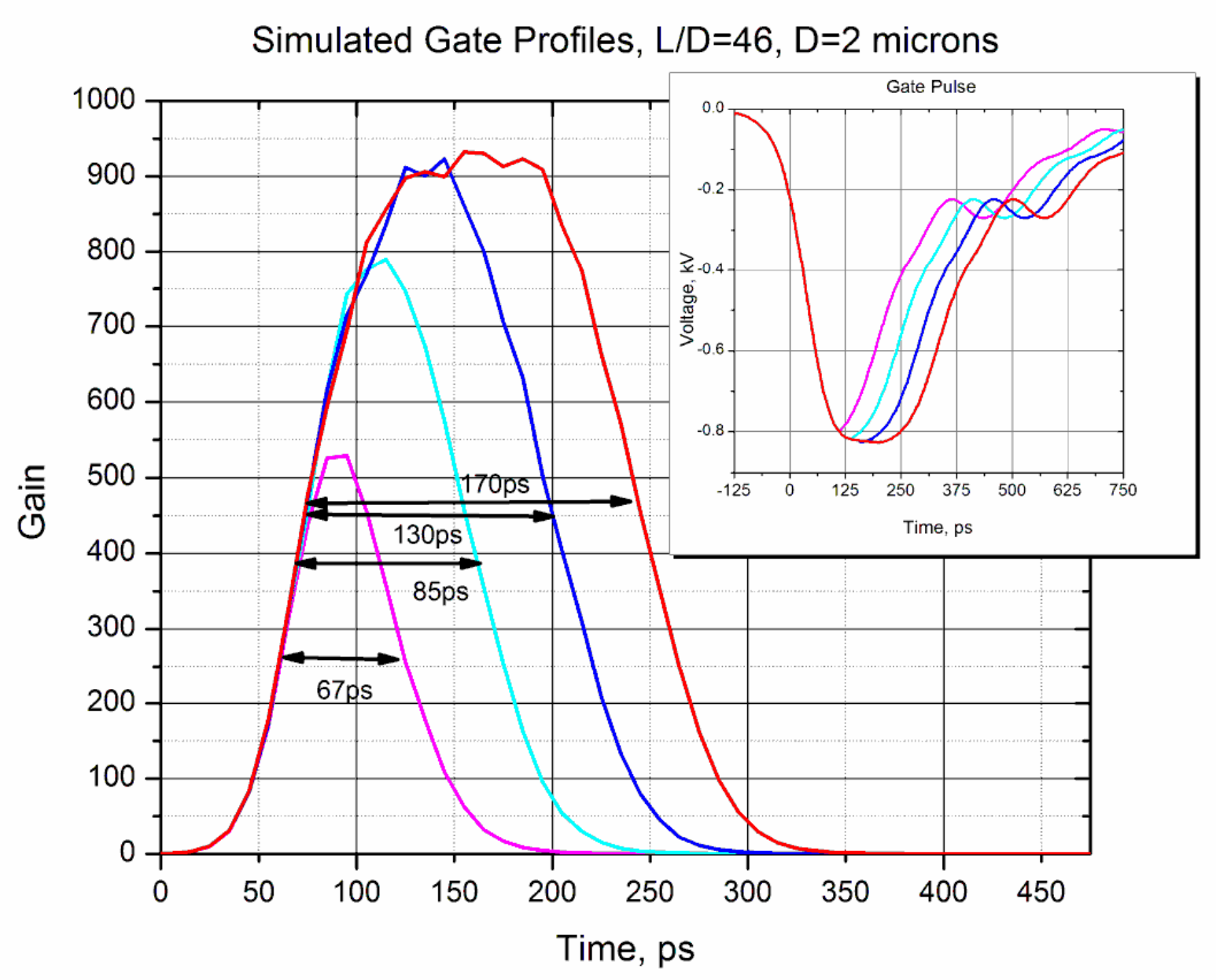




\section{Simulated Gate Profiles}

\section{2-micron MCP, L/D = 60}

The L/D $=60 \mathrm{MCP}$ achieves somewhat shorter gate profile widths, ranging from about 61 ps up to 160 ps.

Gains are smaller, however, being less than 100 for the shortest pulse, and just over 200 for the longest.

The peak gain also is falling off quite rapidly with decreasing voltage pulse width.

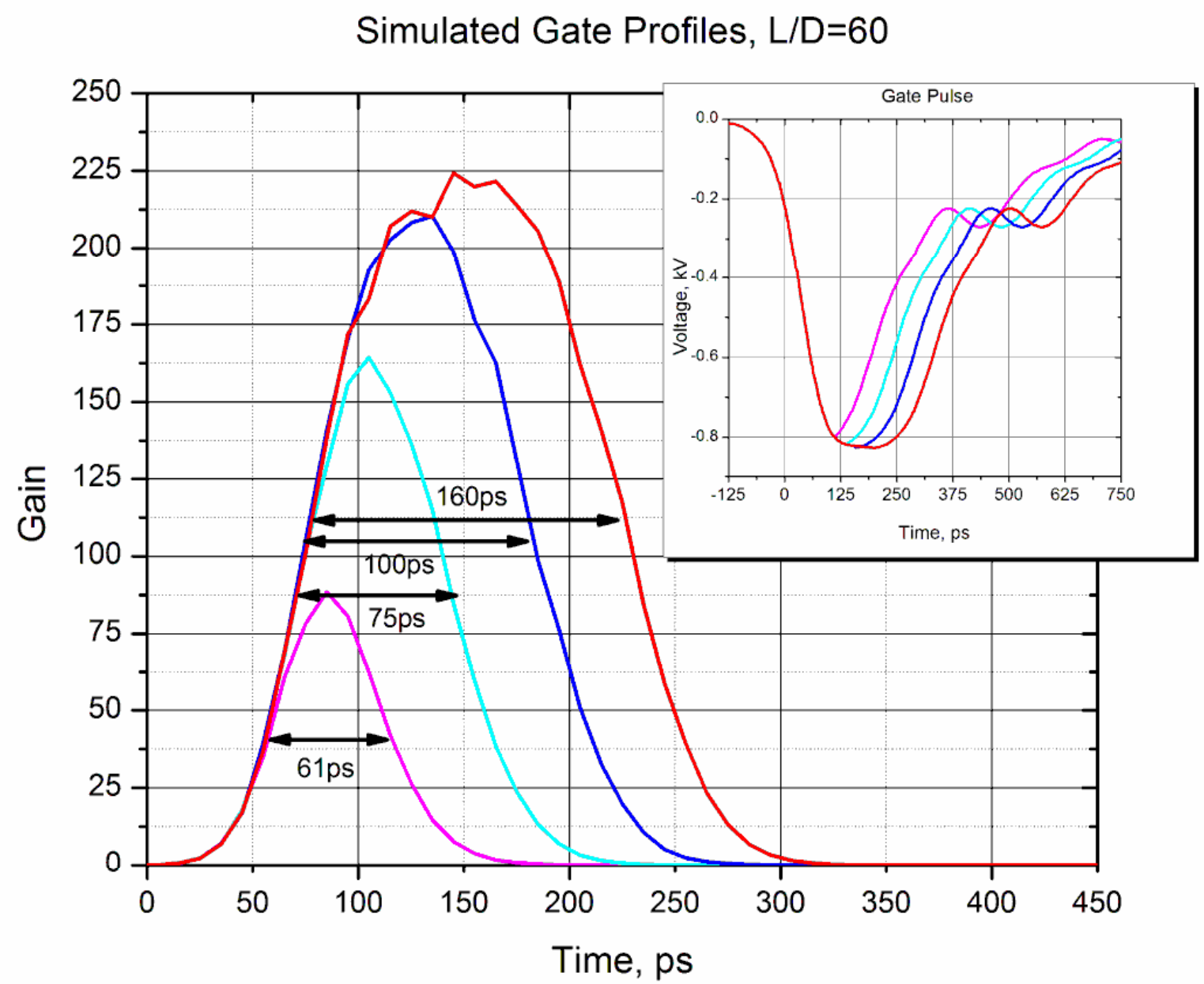




\section{Simulated Gate Profiles}

\section{2-micron MCP, L/D = 80}

The $L / D=80$ MCP achieves shorter still gate profile widths, ranging from about $55 \mathrm{ps}$ up to $130 \mathrm{ps}$. However, gains are very small, however, being less than 10 for all pulses and essentially unity for the shortest. Increasing the voltage (say to 0.9 to $1 \mathrm{kV}$ ) should ameliorate this. As was the case in the $L / D=60$ MCP, peak gains are falling off rapidly with decreasing voltage pulse width.

These simulations seem to indicate that gate profile widths less than 100 ps may be achievable with 2-micron MCPs.

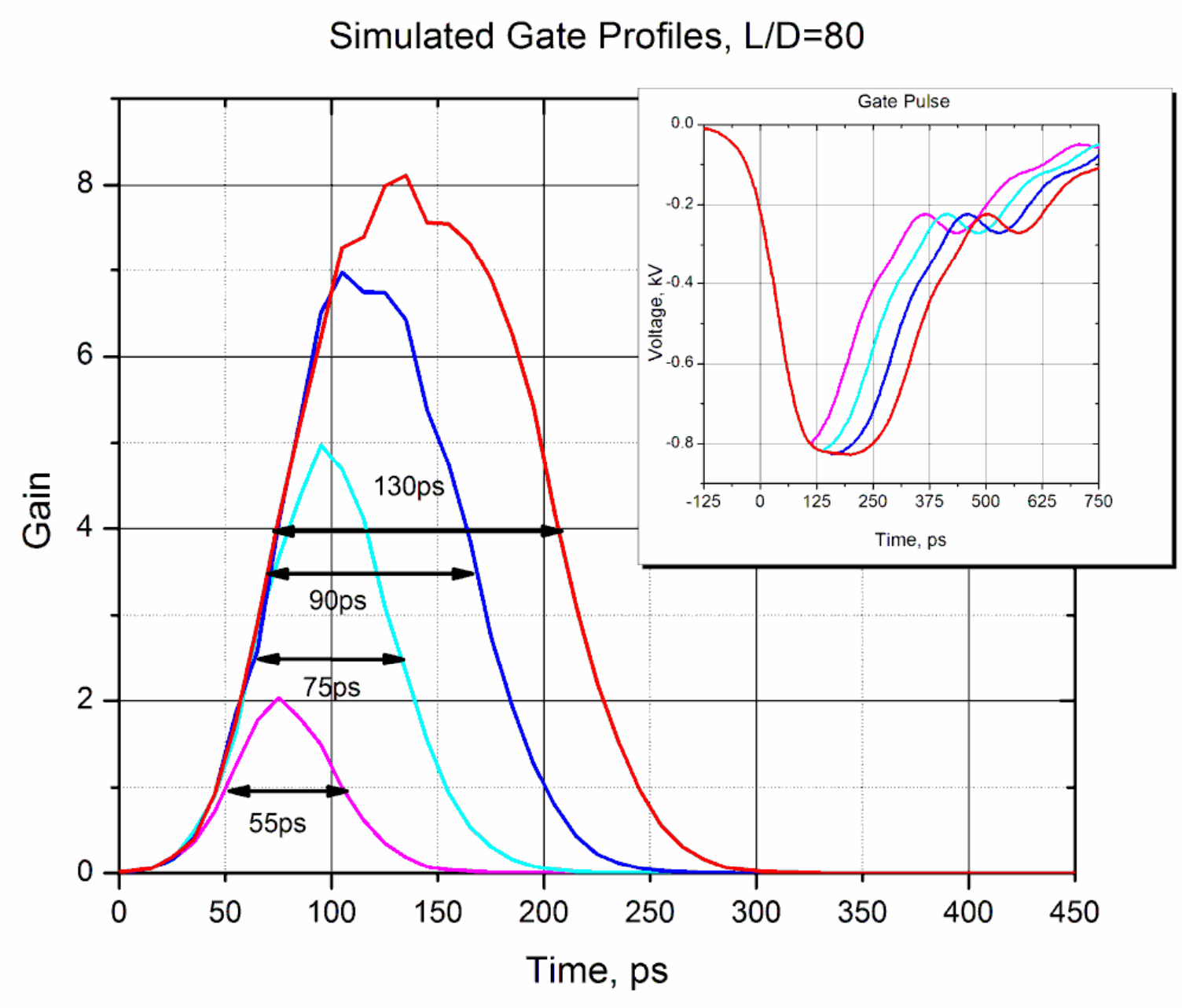




\section{Spatial Resolution Simulations}

\section{0-micron MCP, L/D = 46}

- These spatial resolution simulations were performed for both the 10-micron and 2-micron-pore MCPs.

- The Monte Carlo code extends the trajectories of the output electrons in an applied field between the output of the MCP and a phosphor, taken to be $0.5 \mathrm{~mm}$ away.

- Different MCP-phosphor bias voltages are investigated.

- These plots show results for the 10-micron-pore MCP with different MCP bias angles. The y-direction is taken to be in the direction of the MCP bias angle.

- Spatial resolutions for $4 \mathrm{kV}$ bias voltage are approximately 40 to 50 microns (FWHM of simulated PSF).
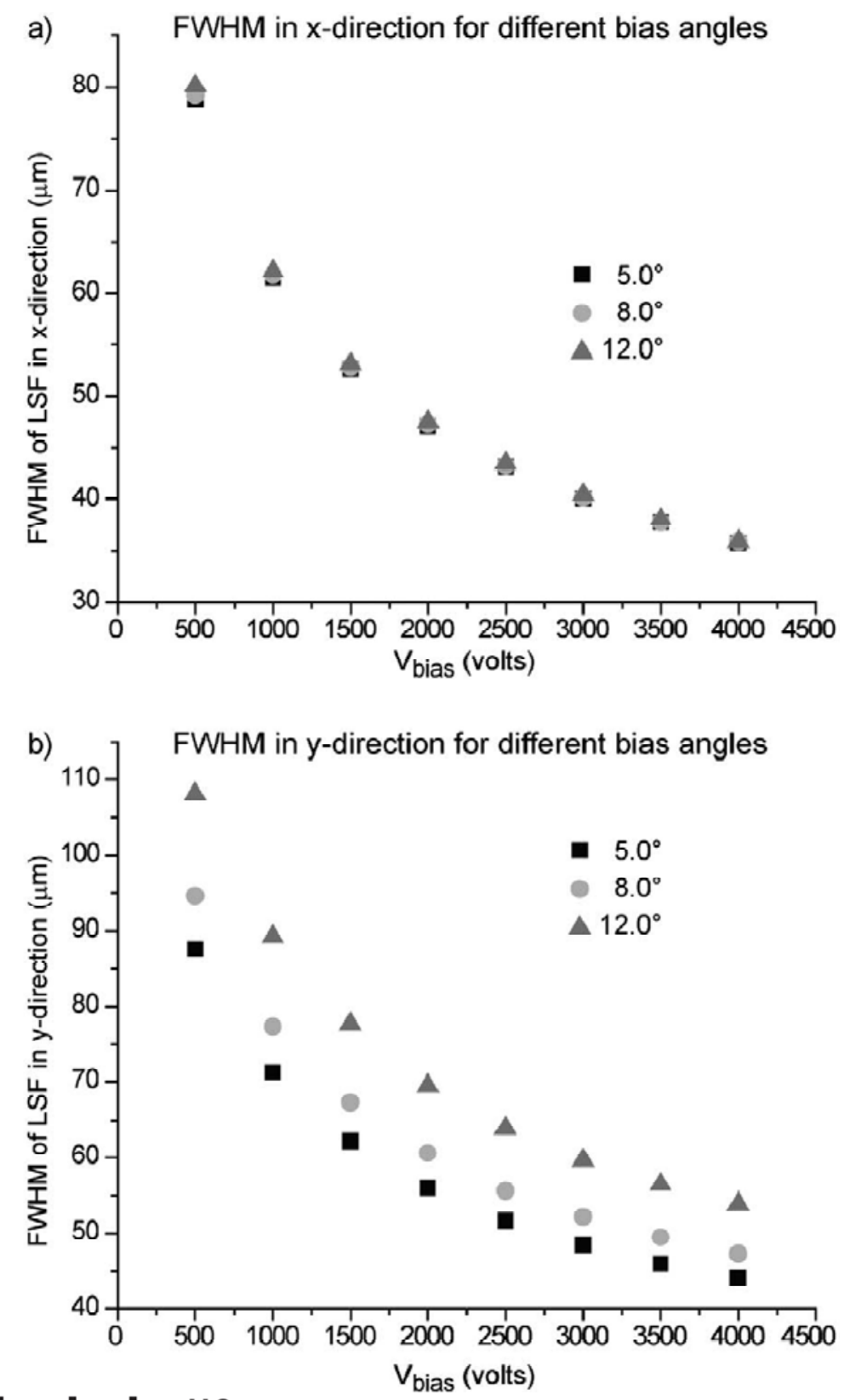


\section{Spatial Resolution Simulations \\ 2-micron MCP, L/D = 60}

- Similar simulations were done for the 2-micronpore MCP. The MCP bias angle is 8 degrees.

- As might be expected, spatial resolutions (FWHM of simulated point spread function) are somewhat smaller, 30 to 40 microns at $4 \mathrm{kV}$ bias voltage.

- Thus, significantly better spatial resolutions are achievable with 2-micron-pore MCPs.

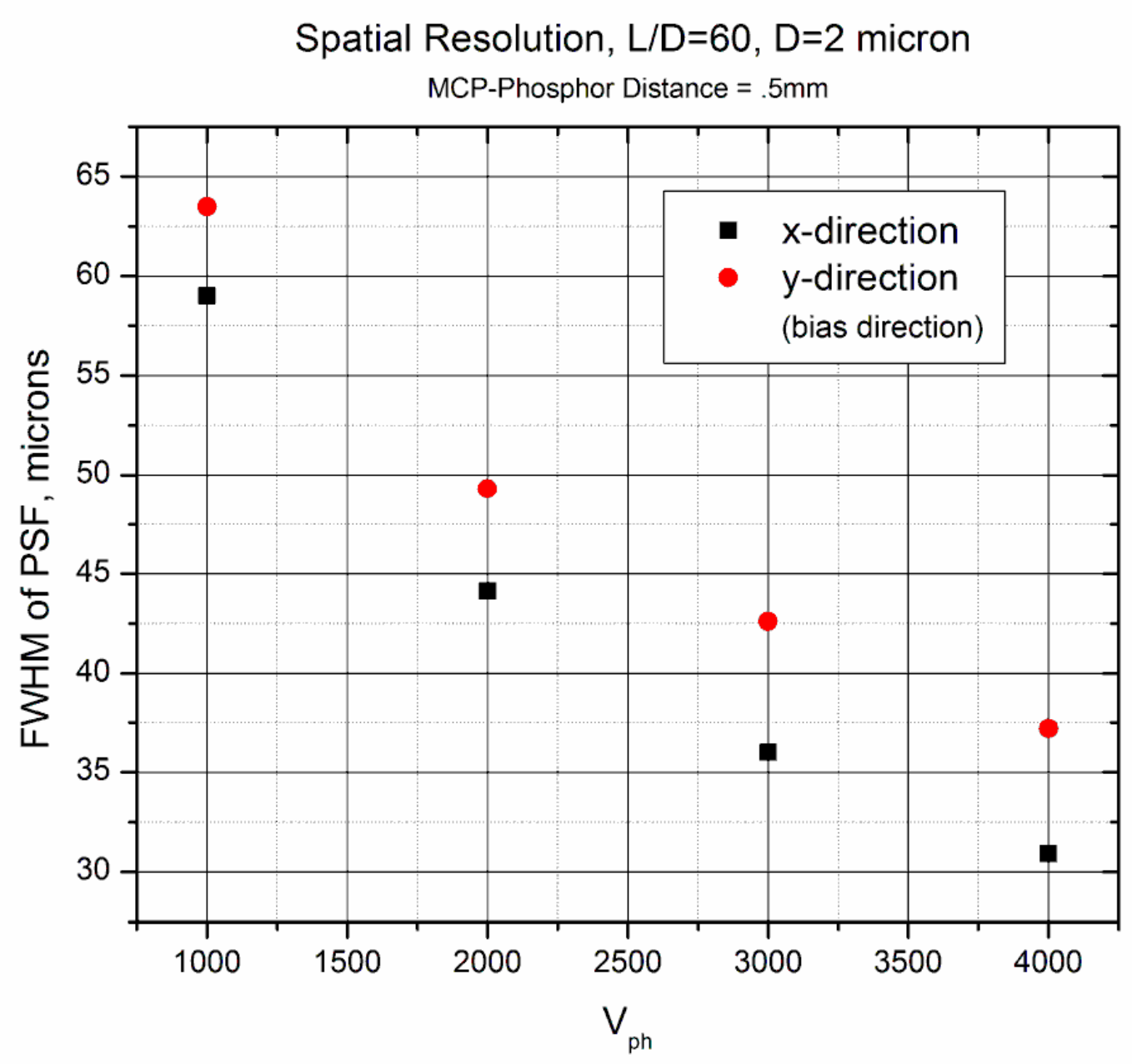




\section{Summary}

We have shown results of a recent simulation study of the potential performance of 2-micron-pore MCPs for fast-gated imaging applications. The results of 2-micron MCP simulations with simulated (and some measured) results from a 10-micron-pore MCP were compared.

We found that for the 2-micron-pore MCPs, gate profiles below 100 ps are potentially achievable given the technology readily available.

Additionally, we found that the simulated spatial resolutions are significantly superior to that achievable with a 10-micron-pore MCP by about $20 \%$.

Thus, small-pore MCPs represent an intriguing possibility for future HEDP imaging diagnostics. 